\title{
Presolar grains in the Solar System: Connections to stellar and interstellar organics
}

\author{
Larry R. Nittler \\ Department of Terrestrial Magnetism, Carnegie Institution of Washington \\ email: lnittler@ciw.edu
}

\begin{abstract}
A small fraction of primitive meteorites and interplanetary dust particles (IDPs) consists of grains of presolar stardust. These grains have extremely unusual isotopic compositions, relative to all other planetary materials, indicating that they condensed in the outflows and explosions of prior generations of stars (Clayton \& Nittler 2004). Identified presolar grain types include silicate, oxide and carbonaceous phases. The latter include graphitic carbon, diamond and SiC. Although many of these phases do not have a direct connection to organic chemistry, this is not true of the graphitic spherules. Many of these, with isotopic compositions indicating an origin in C-rich asymptotic giant branch (AGB) star outflows, have a structure consisting of naonocrystalline cores surrounded by well-graphitized C (Bernatowicz et al. 1996). The cores include isotopically anomalous polycyclic aromatic hydrocarbons (Messenger et al. 1998) and represent a link between molecular chemistry and dust condensation in stellar outflows. Meteorites and IDPs also contain abundant isotopically anomalous organic matter, including distinct organic grains, some of which probably formed in stellar outflows and/or the interstellar medium (ISM) (Busemann et al. 2006, Floss et al. 2004). In some IDPs, deuterium- and ${ }^{15} \mathrm{~N}$-enriched organic matter is closely associated with presolar silicate grains (Messenger et al. 2005, Nguyen et al. 2007), suggesting an association in the ISM prior to Solar System formation.
\end{abstract}

\section{References}

Bernatowicz, T. J., et al. 1996, ApJ, 472, 760

Busemann, H., et al. 2006, Science, 312, 727

Clayton, D. D. \& Nittler, L. R. 2004, ARA\&A, 42, 39

Messenger, S., et al. 1998, ApJ, 502, 284

Messenger, S., et al. 2005, Science, 309, 737

Nguyen, A. N., et al. 2007, LPSC, 38, Abstract \#2332

\section{Discussion}

ZIURYS: You make the point that you have to find an anomalous isotope ratio to say a material is presolar. However, if you look at ${ }^{12} \mathrm{C} /{ }^{13} \mathrm{C}$ ratios in AGB stars, you find ratios from 4 to 100 in the molecular material - UU Omega has a ${ }^{12} \mathrm{C} /{ }^{13} \mathrm{C}$ ratio of 89 , and D Hydra is seventy something. So when you find an odd ratio you can definitely say it comes from outside the Solar System, but just because you see a semi-normal ratio doesn't necessarily mean that it doesn't come from an AGB star.

NiTTLER: Well I disagree with you and the point is that it's not 'sort of normal'. The fact is this organic matter is normal with 'normal' meaning the same as terrestrial carbon standards within 1 or $2 \%$. And you are actually right that you do have values that overlap the terrestrial value, but they are rare in AGB stars. Most of the stuff is very highly 
non-solar and that means the average is nowhere close to solar. So if you have a mixture of materials, it's going to be incredibly coincidental that it has a solar ${ }^{12} \mathrm{C} /{ }^{13} \mathrm{C}$ ratio.

ZiURYs: That's Dave Lambert's study in 1986 and there's far more data these days.

NiTTLER: But the plot you showed in your talk was the same. Every object on that plot was non-solar.

ZIURYS: That is starting with red giants and going into AGBs. I am just saying be cautious since there are carbon AGB stars that have a greater enrichment of ${ }^{12} \mathrm{C}$ to ${ }^{13} \mathrm{C}$.

NiTTLER: I agree. There is a huge range. And the thrust of my argument is that because of the huge range, it's extraordinarily unlikely that you would end up with something the same as terrestrial to within $1 \%$ in any sort of average sense, but you are right that you cannot rule the possibility out. However, if you measure a hundred samples, and they are all terrestrial, it is highly unlikely all one hundred came from an AGB star.

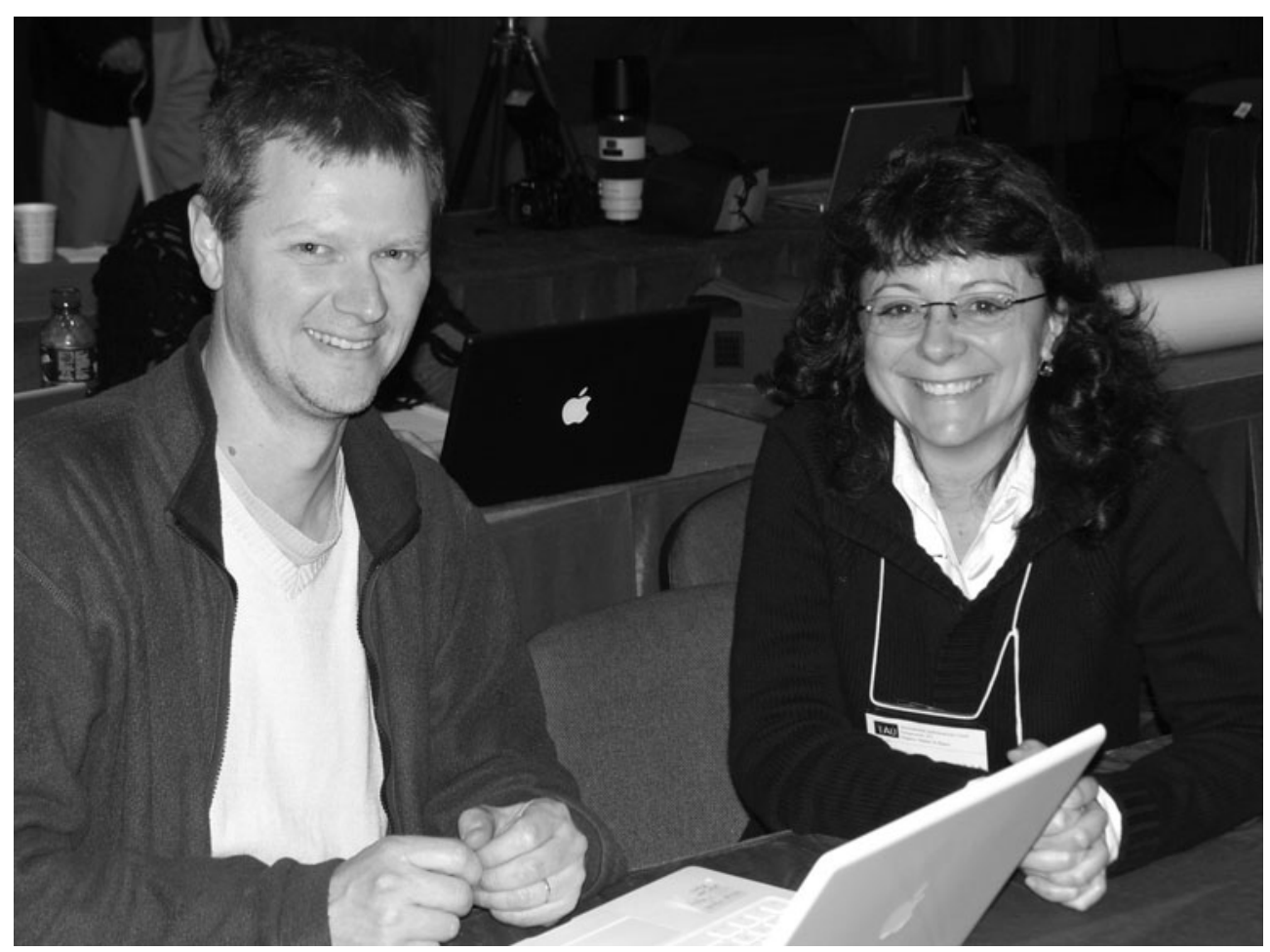

Eric Quirico and Cecelia Ceccarelli (photo by Dale Cruikshank). 